\title{
The impact of diet on the composition and relative abundance of rumen microbes in goat
}

\author{
Kaizhen Liu' ${ }^{1}$, Qin $\mathrm{Xu}^{1}$, Lizhi Wang ${ }^{1, *}$, Jiwen Wang ${ }^{1}$, Wei Guo ${ }^{1}$, and Meili Zhou ${ }^{1}$
}

* Corresponding Author: Lizhi Wang Tel: +86-138-82438306, Fax: +86-835-2885065,

E-mail: wanglizhi08@aliyun.com

'Institute of Animal Nutrition, Sichuan Agricultural University, Ya'an 625014, China

Submitted May 5, 2016; Revised Jul 11, 2016; Accepted Aug 8, 2016
Objective: This experiment was conducted to explore the impact of diet on the ruminal microbial community in goats.

Methods: Twelve goats were divided into two groups and fed complete feed (CF) or all forage (AF) diet. The total microbial DNAs in the rumen liquid were extracted. The V4 region of microbial 16S rRNA genes was amplified and sequenced using high-throughput. Information of sequences was mainly analyzed by QIIME 1.8.0.

Results: The results showed that Bacteroidetes and Firmicutes were the most predominant microbial phyla in the rumen of all goats. At genus level, the abundance of fiber-digesting bacteria such as Ruminococcus and Lachnospiracea incertae sedis was significantly higher in $\mathrm{AF}$ than that in CF, while the levels of fat-degrading bacterium Anaerovibrio and proteindegrading bacterium Pseudomonas were opposite. The core shared genera, Prevotella and Butyrivibrio were widespread in the rumen of goats and no significant difference was observed in relative abundance between groups.

Conclusion: We concluded that the richness of fiber-, protein-, and fat-digesting bacteria was affected by diet and tended to increase with the rise of their corresponding substrate contents in the ration; some bacteria shared by all goats maintained stable despite the difference in the ration, and they might be essential in maintaining the normal function of rumen.

Keywords: Goat; Diet; Rumen Microbe; Diversity; High-throughput Sequencing

\section{INTRODUCTION}

Compared with monogastric animals, the ruminants have a more specialized stomach composed of four separate chambers and the rumen is the largest. In the rumen, there are high-density microorganisms, such as bacteria, archaea, fungi, and protozoa. These microorganisms play a vital role in maintaining the normal digestion and absorption in the host. They can digest the fibers into monosaccharides and disaccharides and then convert them into fatty acids, which can be absorbed and used by the host $[1,2]$.

It was previously reported that ruminal Prevotella in sheep was more diverse with the increase of fiber content in ration [3], the abundance of Bacteroides in the rumen of steers decreased with the increase of neutral detergent fiber in diets [4], and the rumen Fibrobacteraceae concentration was higher in cows fed with total mixed ration than the free-range cows [5]. These previous researches suggested that diets had significant impact on ruminal microbial community. However, more experimental evidence is needed to confirm this postulation. Moreover, many kinds of ruminants including cows, cattle, buffalo, yak and sheep have been used as experimental subjects to determine the microbial diversity in rumen $[2,6,7]$, while goats are rarely used in studies. And, the previous goat-related studies [8,9] mainly used conventional methods, such as molecular fingerprint, to evaluate the ruminal microbial community. These methods could 
only identify the dominant species instead of the complete composition of the microorganisms in sample when microbial diversity was investigated [10]. Therefore, the impact of changes in diets on the microflora in goat still remains unclear. Highthroughput sequencing is a novel technology, which can process millions of sequenced reads at the same time and obtain the biological information of most microorganisms in sample through deep sequencing, has been recently applied in microbial ecology [11]. In the present study, high-throughput sequencing was applied to investigate the impact of the difference in diet on the microbial structure and composition of the rumen in goats.

\section{MATERIALS AND METHODS}

The animal experimental procedures were approved by the Committee of Laboratory Animal Welfare and Ethics of the Sichuan Agricultural University.

\section{Animal experiment and rumen sampling}

Twelve castrated 1-yr-old hybrid goats (Jianchang black goat and Boer goat crossbred) with an average body weight of $25 \pm 2 \mathrm{~kg}$ were used in this study. They were divided into two groups ( 6 goats per group) according to their age and weight and assigned to two treatments, CF group offered complete feed and AF group offered all-forage diet. The chemical composition of diets for $\mathrm{CF}$ and AF are shown in Supplementary Table S1a and S1b, respectively. The goats were kept in separate cages with free access to water. They were fed a restricted dry matter at the level of 3\% of their body in equal amounts at 09:00 and 17:00 h. After 20 days of adaption, rumen contents $(50 \mathrm{~mL})$ were collected from each goat using a stomach tube before morning feeding. The rumen contents were strained by four layers of gauze and the rumen fluid was aliquoted into $10 \mathrm{~mL}$ centrifuge tubes. The samples were sealed and stored at $-80^{\circ} \mathrm{C}$ until DNA extraction.

\section{Polymerase chain reaction amplification and sequencing} Total DNA of samples was extracted using DNA Stool Kit (TIANGEN, Beijing, China). The universal prokaryote primer set, 515F and 806R [12], were used to amplify the V4 region of $16 \mathrm{~S}$ rRNA. Three replicate polymerase chain reactions (PCRs) were performed for each DNA sample. The PCR reaction volume was $20 \mu \mathrm{L}$, including $0.4 \mu \mathrm{L}$ FastPfu Polymerase (Transgen, Bio Inc., Beijing, China), $4 \mu \mathrm{L} 5 \times$ FastPfu buffer, $0.4 \mu \mathrm{L}$ of each forward and reverse primers $(5 \mu \mathrm{M}), 2 \mu \mathrm{L}$ deoxynucleoside triphosphates mix (dNTP, $2.5 \mathrm{mM}$ ) and template, DNA $10 \mathrm{ng}$. The $\mathrm{ddH}_{2} \mathrm{O}$ was added to a final volume of $20 \mu \mathrm{L}$. The PCR was performed using a thermal cycler Model (ABI GeneAmp 9700, Applied Biosystems Inc., San Diego, CA, USA) with the thermal cycling conditions: initial denaturation of $2 \mathrm{mi}$ at $95^{\circ} \mathrm{C}, 30$ cycles of denaturation at $95^{\circ} \mathrm{C}$ for $30 \mathrm{~s}$, annealing at $56^{\circ} \mathrm{C}$ for $30 \mathrm{~s}$, extension at $72^{\circ} \mathrm{C}$ for $30 \mathrm{~s}$, final extension at $72^{\circ} \mathrm{C}$ for $5 \mathrm{~min}$ and hold at $10^{\circ} \mathrm{C}$. The PCR products from the same sample were mixed together and purified using the AxyPrep DNA Gel Extraction kit (AXYGEN, Silicon Valley, MA, USA). Purified amplicons were paired-end sequenced $(2 \times 150 \mathrm{nt}$ reads) on Illumina MiSeq platform at the MaJorbio Co. of ShangHai (ShangHai, China), as described before [13].

\section{Sequence analysis}

The primer sequences and multiplexing barcodes were trimmed. The poor/low quality sequences including those with uncertain nucleotides, continuous three nucleotides with $Q$ value less than 20 , unmatched barcode sequences were discarded. The obtained sequences were clustered using Uparse7.0 into operational taxomonic units (OTUs) based on 97\% similarity. Chimeras were removed using UCHIME 4.2.4 [14]. The most abundant sequence was selected as the representative for each OTU, and then aligned against the Greengenes database (http://greengenes. lbl.gov) using PyNAS [15]. Representative sequences were taxonomically classified using the ribosomal database project (RDP) taxonomic database. Calculations for rarefraction curves, the estimated maximum number of OTUs, Shannon-wiener index and chaoI index were performed using QIIME version 1.8.0 [16]. The current $\%$ coverage was calculated by dividing the observed number of OTUs by the maximum number of OTUs estimated. The Weighted UniFrac distance metric was calculated to compare the phylogenetic similarity between the bacterial communities. Principal co-ordinate analysis (PCoA) cluster plot was performed using $\mathrm{R}$ version 3.1.0 for visualization. All Illumina sequences data in this study were deposited to the SRA of the NCBI database under BioProject PRJNA290546.

\section{Statistical analysis}

The relative abundance of microbes was log-transformed [17] and the unpaired two-tailed t-test was performed using the SPSS Statistics software v. 19.0 (IBM, Armonk, NY, USA) to assess whether there was a significant difference between CF and AF. Significance level was set at $\mathrm{p}<0.05$.

\section{RESULTS}

\section{Data depth and alpha diversity}

A total of 251,229 sequences were generated after quality control with a mean of 20,809 $\pm 6,497$ (mean \pm standard deviation [SD], $\mathrm{n}=12$ ) per sample. These sequences were totally clustered into 13,615 OTUs with a mean of $1,983 \pm 768($ mean $\pm S D, n=12)$ per sample. There were 6,242 OTUs in CF group and 9,510 OTUs in AF group. The difference in the average number of OTUs between the two groups was not significant $(\mathrm{p}>0.05)$ (Supplementary Figure S1). The rarefaction curve (Figure 1) showed that most samples were nearly asymptotic, which indicated that the depth of sequencing in the present study could cover most of the microorganisms in the sample. The average of the 


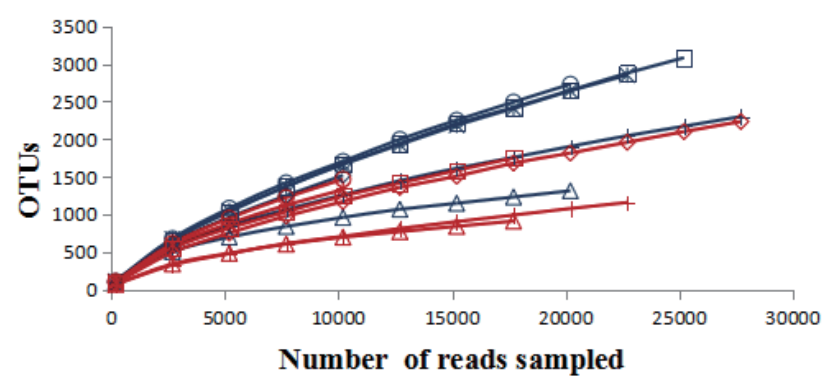

Figure 1. The rarefaction analysis was conducted at $97 \%$ sequence identity, and each curve represented one individual rumen sample of goats $(n=12)$. The blue curves: individuals fed at a completed feed (CF) diet; the red curves: individuals fed at all forage (AF) diet.

estimated maximum number of OTUs and current \% coverage of each sample at $97 \%$ similarity is 3,376 and $65.3 \%$, respectively. Alpha diversity is presented in Table 1. Shannon-Wiener index, Chaol index and the observed-species were all higher in $\mathrm{CF}$ than that in AF. However, the differences were not statistically significant $(\mathrm{p}>0.05)$.

\section{Rumen bacterial composition}

The sequences in the present experiment were finally annotated as bacteria $(99.11 \% \pm 40.25 \%)$, archaea $(0.20 \% \pm 0.23 \%)$ and the unassigned microorganisms $(0.69 \% \pm 0.39 \%)$. The bacteria kingdom is composed of 23 phyla, of which 22 were identified in CF group and 19 in AF group. The phyla with a relative abundance greater than 1\% were Bacteroides, Firmicutes, Proteobacteria, Tenericutes, Verrucomicrobia, and Lentisphaerae in CF, Bacteroides, Firmicutes, Proteobacteria, and Verrucomicrobia in AF. The abundance of Firmicutes in CF was significantly lower ( $p=0.027$ ) than that in AF. In contrast, the abundance of Proteobacteria was extremely significantly lower $(\mathrm{p}<0.001)$ in AF than that in CF. There was no significant difference in the abundance of other phyla in the two groups (Table 2). Moreover, the bacterial sequences not affiliated with any known phylum were annotated as unclassified bacteria, which made up $2.99 \% \pm 2.67 \%$ and $3.56 \% \pm 4.07 \%$ of the total microbiome in CF and AF group, respectively. At genus level, there were 15 members which differed significantly $(\mathrm{p}<0.05)$ in abundance between CF and AF (Table 3). They mainly came from the phylums Firmicutes and Proteobacteria.

\section{Shared genera}

Table 1. The alpha diversity index (calculated at a depth of 10,200 sequences) of bacterial communities from goats fed at a completed feed (CF) or all forage (AF) diet $($ mean $\pm S D, n=12)$

\begin{tabular}{lccc}
\hline Groups & Shannon & Chaol & The observed-species \\
\hline AF & $6.98 \pm 0.66$ & $3,324.00 \pm 1,653.29$ & $1,244.17 \pm 395.87$ \\
CF & $7.23 \pm 0.69$ & $3,755.36 \pm 1,107.96$ & $1,322.00 \pm 334.69$ \\
p value & 0.55 & 0.61 & 0.72 \\
\hline
\end{tabular}

Table 2. The bacterial compositions and their relatively abundance at the phylum level in the rumen of goats fed a completed feed (CF) or all forage (AF) diet

\begin{tabular}{|c|c|c|c|}
\hline \multirow{2}{*}{ Phylum } & \multicolumn{2}{|c|}{ Log-transformed proportion values } & \multirow{2}{*}{$p$ value } \\
\hline & CF & $\mathrm{AF}$ & \\
\hline Bacteroidetes & $1.74^{11} \pm 1.06^{2)}$ & $1.68 \pm 1.01$ & 0.387 \\
\hline Firmicutes & $1.41 \pm 0.89$ & $1.63 \pm 1.15$ & 0.027 \\
\hline Proteobacteria & $0.86 \pm 0.52$ & $0.08 \pm-0.06$ & 0.000 \\
\hline Unclassified & $0.48 \pm 0.43$ & $0.55 \pm 0.61$ & 0.967 \\
\hline Tenericutes & $0.44 \pm 0.67$ & $-0.51 \pm-0.54$ & 0.265 \\
\hline Verrucomicrobia & $0.40 \pm 0.34$ & $0.03 \pm 0.0 .1$ & 0.169 \\
\hline Lentisphaerae & $0.31 \pm 0.23$ & $-0.26 \pm-0.75$ & 0.055 \\
\hline Spirochaetes & $-0.24 \pm-0.53$ & $-0.18 \pm-0.34$ & 0.683 \\
\hline TM7 & $-0.37 \pm-0.39$ & $-0.47 \pm-0.67$ & 0.989 \\
\hline SR1 & $-2.05 \pm-2.08$ & $-0.46 \pm-0.37$ & 0.083 \\
\hline Other & $-0.91 \pm-1.03$ & $-1.35 \pm-1.46$ & 0.100 \\
\hline Chloroflexi & $-1.04 \pm-0.88$ & $-1.70 \pm-1.86$ & 0.218 \\
\hline Fibrobacteres & $-1.25 \pm-1.26$ & $-1.27 \pm-1.12$ & 0.965 \\
\hline Synergistetes & $-1.09 \pm-1.00$ & $-1.68 \pm-1.58$ & 0.185 \\
\hline Elusimicrobia & $-1.43 \pm-1.31$ & $-1.33 \pm-1.15$ & 0.798 \\
\hline Actinobacteria & $-1.59 \pm-1.40$ & $-1.48 \pm-1.66$ & 0.281 \\
\hline Planctomycetes & $-1.55 \pm-1.32$ & $-1.97 \pm-1.95$ & 0.409 \\
\hline Armatimonadetes & $-1.84 \pm-1.61$ & $-2.14 \pm-2.14$ & 0.496 \\
\hline Acidobacteria & $-2.43 \pm-2.04$ & $0^{3)}$ & 0.341 \\
\hline Gemmatimonadetes & $-2.43 \pm-2.04$ & 0 & 0.341 \\
\hline Cyanobacteria & 0 & $-2.87 \pm-2.68$ & 0.147 \\
\hline Fusobacteria & $-3.15 \pm-2.76$ & 0 & 0.341 \\
\hline Nitrospira & $-3.13 \pm-2.74$ & 0 & 0.341 \\
\hline
\end{tabular}

1) Log-transformed proportion values of the average value of six goats.

2) Log-transformed proportion values of the standard deviation value of six goats.

3) Not detected.

There were 33 shared genera within the $\mathrm{CF}, 30$ within the AF and 26 shared by both groups (Table 4). Eight shared genera in CF were greater than $1 \%$ in abundance, including Prevotella, Pseudomonas, Subdivision5 genera incertae sedis, Ruminococcus, Butyrivibrio, Succiniclasticum, Clostridium IV, and Vampirovibrio. Seven shared genera in AF were greater than $1 \%$ in abundance, including Prevotella, Ruminococcus, Lachnospiracea incertae sedis, Butyrivibrio, Succiniclasticum, Saccharofermentans, and Subdivision5 genera incertae sedis. There were eight genera shared by both groups with the average abundance greater than $1 \%$, including Prevotella, Ruminococcus, Butyrivibrio, Succiniclasticum, Clostridium IV, Lachnospiracea incertae sedis, Saccharofermentans, and Subdivision5 genera incertae sedis.

\section{Weighted unifrac distance analysis}

The weighted uniFrac distance matrices were calculated according to the types and abundance of OTU of each sample. Similarity analysis based on the distance matrices showed that the similarity between $\mathrm{CF}$ and $\mathrm{AF}$ was $61.99 \%$. The intra-group similarity of $\mathrm{CF}$ and $\mathrm{AF}$ was $62.76 \%$ and $67.94 \%$, respectively. The PCoA plot (Figure 2) showed that four samples in CF clustered together while the remaining two were far from them, and samples in AF were evenly distributed in the cluster. All samples tended to cluster together in accordance with their own ration treatment. 
Table 3. The 15 genera with significantly different abundance in the rumen of goats fed at a completed feed (CF) or all forage (AF) diet ${ }^{1)}$

\begin{tabular}{|c|c|c|c|c|}
\hline \multirow{2}{*}{ Phylum } & \multirow{2}{*}{ Genus } & \multicolumn{2}{|c|}{ Log-transformed proportion values } & \multirow{2}{*}{$p$ value } \\
\hline & & $\mathrm{CF}$ & AF & \\
\hline \multirow[t]{4}{*}{ Firmicutes } & Lachnospiracea_incertae_sedis & $-0.22^{2)} \pm-0.59^{3)}$ & $0.89 \pm 0.50$ & 0.000 \\
\hline & Flavonifractor & $-0.04 \pm-2.23$ & $-3.16 \pm-2.77$ & 0.007 \\
\hline & (Phylum) Firmicutes & $0.16 \pm-0.11$ & $0.50 \pm 0.04$ & 0.013 \\
\hline & Eubacterium & $0^{4)}$ & $-2.38 \pm-2.43$ & 0.021 \\
\hline \multirow[t]{6}{*}{ Proteobacteria } & (Family) Enterobacteriaceae (other) & $0.10 \pm-0.12$ & $-1.10 \pm-1.22$ & 0.038 \\
\hline & Sphingomonas & $-2.14 \pm-1.91$ & $-1.56 \pm-1.87$ & 0.023 \\
\hline & (Family)Comamonadaceae & $-3.13 \pm-2.74$ & $-2.31 \pm-2.48$ & 0.022 \\
\hline & Pseudomonas & $-0.11 \pm-0.08$ & $-1.10 \pm-1.40$ & 0.000 \\
\hline & (Family)Enterobacteriaceae (unlassfied) & $-1.72 \pm-1.82$ & $-2.48 \pm-2.28$ & 0.003 \\
\hline & Enterobacter & $-1.94 \pm-2.01$ & $-2.75 \pm-2.51$ & 0.044 \\
\hline
\end{tabular}

\section{DISCUSSION}

In this study, the predominant phyla in the rumen of goats were Bacteroidetes and Firmicutes (Table 2), which accounted for $80.3 \%$ and $90.7 \%$ of the total microbial abundance in CF and AF, respectively. This was consistent with many past studies in herbivores [18-20]. At phylum level, Firmicutes had significantly lower abundance in CF (25.87\%) than that in AF (42.80\%). We explored the reasons at a deeper classification and discovered that four genera within phylum Firmicutes had significantly lower abundance in $\mathrm{CF}$ than that in $\mathrm{AF}$, and 3 of them including Lachnospiracea incertae sedis, (phylum) Firmicutes and Ruminococcus were worth of noting because of their high relative abundance. These differences in abundance might due to the different fiber content in diets (Supplementary Table S1). Ruminococcus is composed of two strong fiber-digesting bacteria species, Ruminococcus albus and Ruminococcus flavefaciens, which can produce a large amount of Cellulases and hemicellulases [21]. The higher abundance of Ruminococcus corresponding to the higher fiber content probably came from the effect of substrate-induced. It was reported that rumen Lachnospiracea incertae sedis functioned synergistically with Fibrobacter succinogenes in the fiber digestion [22]. However, Lachnospiracea incertae sedis was poorly characterized phylogenetically and physiologically because of the difficulty of isolation [23]. Therefore, its role in fiber digestion requires further research. The function of the unassigned genus in Firmicutes (phylum) is unknown. We assumed that it might be a novel microorganism involved in fiber digestion. Further work is needed to confirm this speculation.
In the present study, phylum Proteobacteria had significantly lower abundance in $\mathrm{AF}(1.19 \%)$ than that in $\mathrm{CF}$ (7.24\%). It was reported that Proteobacteria had the highest abundance in the rumen of newborns ( 1 to $3 \mathrm{~d}$ ) fed with colostrum and 2-monthold calves fed with milk-supplemented ration, and its abundance sharply decreased in the rumen of the 6-month-old and 2-yrold cows [2]. It is obvious that the fiber content was higher while the protein content was lower in the ration of the 6-month-and 2 -yr-old cows compared with the ration of the calves. Similarly, the protein content was lower while neutral detergent fiber and acid detergent fiber contents were substantially higher in $\mathrm{AF}$ than that in CF (Supplementary Table S1). Therefore, it might be a reasonable speculation that the abundance of Proteobacteria would decrease with the increase of fiber content and was positively correlated with the protein content in the ration. Analysis at the genus level seemed to support this speculation, as the major genus within phylum Proteobacteria whose abundance was significantly higher in $\mathrm{CF}$ than that in AF was Pseudomonas. It was reported that many strains of Pseudomonas could produce keratinase, which possessed strong protein-degrading function [24].

At genus level, Prevotella was shared by all samples and had the highest abundance both in CF and AF in our study (Table 4), which was in accordance with previous studies in other ruminants $[3,9]$. Analyzing the previous experiments, we found that the abundance of Prevotella did not always demonstrate regulated changes with the alteration of ration. For example, Huo et al [25] found that the abundance of Prevotella in the rumen from hay-fed goats was higher than concentrate-fed animals. However, Bekele et al [3] found that although the abundance of Prevotella 
Table 4. There were 33-shared genera in the rumen of goats fed at a completed feed (CF) diet and 30 fed all forage (AF) diet as well as 26 shared by all goats

\begin{tabular}{|c|c|c|c|c|}
\hline \multirow{2}{*}{ Phylum } & \multirow{2}{*}{ Genus } & \multicolumn{3}{|c|}{ Log-transformed proportion values } \\
\hline & & $\mathrm{CF}$ & $\mathrm{AF}$ & All goats \\
\hline \multirow[t]{5}{*}{ Bacteroidetes } & Prevotella & $1.41^{1)} \pm 1.17^{21}$ & $1.35 \pm 0.75$ & $1.38 \pm 1.03$ \\
\hline & Paraprevotella & $-0.16 \pm-0.18$ & $-0.40 \pm-0.61$ & $-0.26 \pm-0.30$ \\
\hline & Hallella & $-0.31 \pm-0.23$ & $-0.07 \pm 0.10$ & $-0.17 \pm-0.16$ \\
\hline & Rikenella & $-0.97 \pm-1.12$ & $-0.52 \pm-0.40$ & $-0.69 \pm-0.54$ \\
\hline & Barnesiella & $-1.24 \pm-1.37$ & Unshared genus & Unshared genus \\
\hline \multirow[t]{17}{*}{ Firmicutes } & Ruminococcus & $0.14 \pm-0.27$ & $0.53 \pm 0.29$ & $0.37 \pm 0.23$ \\
\hline & Butyrivibrio & $0.57 \pm 0.69$ & $0.52 \pm 0.24$ & $0.55 \pm 0.54$ \\
\hline & Succiniclasticum & $0.15 \pm 0.32$ & $0.28 \pm 0.40$ & $0.22 \pm 0.35$ \\
\hline & Clostridium IV & $0.11 \pm-0.21$ & $-0.11 \pm-0.40$ & $0.01 \pm-0.25$ \\
\hline & Lachnospiracea_incertae_sedis & $-0.22 \pm-0.58$ & $0.89 \pm 0.50$ & $0.62 \pm 0.63$ \\
\hline & Saccharofermentans & $-0.09 \pm-0.00$ & $0.22 \pm 0.08$ & $0.10 \pm-0.00$ \\
\hline & Oscillibacter & $-0.33 \pm-0.19$ & $-0.64 \pm-0.78$ & $-0.46 \pm-0.33$ \\
\hline & Pseudobutyrivibrio & $-0.33 \pm-0.43$ & $-0.81 \pm-1.06$ & $-0.51 \pm-0.52$ \\
\hline & Selenomonas & $-0.61 \pm-0.54$ & $-1.37 \pm-1.54$ & $-0.84 \pm-0.65$ \\
\hline & Sporobacter & $-0.84 \pm-0.99$ & $-0.52 \pm-0.52$ & $-0.65 \pm-0.64$ \\
\hline & Anaerovorax & $-0.90 \pm-0.25$ & $-0.82 \pm-0.92$ & $-0.86 \pm-1.05$ \\
\hline & Clostridium XIVb & $-0.99 \pm-1.03$ & $-1.02 \pm-1.42$ & $-1.01 \pm-1.16$ \\
\hline & Acetivibrio & $-1.10 \pm-1.20$ & $-0.96 \pm-1.04$ & $-1.02 \pm-1.11$ \\
\hline & Anaerovibrio & $-1.10 \pm-1.35$ & Unshared genus & Unshared genus \\
\hline & Roseburia & $-1.21 \pm-1.17$ & $-0.61 \pm-0.27$ & $-0.81 \pm-0.42$ \\
\hline & Moryella & $-1.47 \pm-1.38$ & Unshared genus & Unshared genus \\
\hline & Clostridium XIVa & Unshared genus & $-2.72 \pm-2.50$ & Unshared genus \\
\hline \multirow[t]{7}{*}{ Proteobacteria } & Pseudomonas & $0.11 \pm 0.07$ & $-0.10 \pm-1.42$ & $-0.17 \pm 0.01$ \\
\hline & Vampirovibrio & $0.05 \pm 0.08$ & $-0.22 \pm-0.13$ & $-0.06 \pm-0.00$ \\
\hline & Succinivibrio & $-2.45 \pm-2.06$ & Unshared genus & Unshared genus \\
\hline & Desulfovibrio & $-0.76 \pm-0.51$ & Unshared genus & Unshared genus \\
\hline & Acinetobacter & $-0.95 \pm-0.79$ & Unshared genus & Unshared genus \\
\hline & Stenotrophomonas & $-1.68 \pm-1.67$ & $-1.82 \pm-2.23$ & $-1.74 \pm-1.82$ \\
\hline & Sphingomonas & Unshared genus & $-1.56 \pm-1.87$ & Unshared genus \\
\hline Verrucomicrobia & Subdivision5_genera_incertae_sedis & $-1.27 \pm-1.06$ & $-1.81 \pm-1.62$ & $-1.46 \pm-1.20$ \\
\hline Tenericutes & Anaeroplasma & $-0.08 \pm-0.49$ & $-0.51 \pm-0.54$ & $-0.24 \pm-0.39$ \\
\hline Lentisphaerae & Victivallis & Unshared genus & $-0.28 \pm-0.71$ & Unshared genus \\
\hline TM7 & TM7 genera incertae sedis & $-0.37 \pm-0.39$ & $-0.47 \pm-0.67$ & $-0.42 \pm-0.51$ \\
\hline \multirow[t]{2}{*}{ Spirochaetes } & Treponema & $-0.38 \pm-0.66$ & $-0.38 \pm-0.41$ & $-0.38 \pm-0.52$ \\
\hline & Spirochaeta & $-0.84 \pm-0.81$ & $-0.65 \pm-0.86$ & $-0.73 \pm-0.84$ \\
\hline Euryarchaeota & Methanobrevibacter & $-1.19 \pm-1.31$ & Unshared genus & Unshared genus \\
\hline SR1 & SR1 genera incertae sedis & Unshared genus & $-0.46 \pm-0.37$ & Unshared genus \\
\hline
\end{tabular}

1) Log-transformed proportion values of the average value of each sample.

2) Log-transformed proportion values of the standard deviation value of each sample.

in the rumen of sheep did not differ significantly in the various ration groups, it showed an increasing trend in the concentratefed group compared to the roughage group, which was consistent with our results. In our research, although the abundance of Prevotella was numerically higher in the CF, the difference was not significant between the two groups. There are many species among Prevotella which are involved in the degradation of hemicelluloses, pectin, starch, protein, respectively [26]. Due to the various Prevotella species and their different substrate preference, the changes in ration might not always induce regular variation in the abundance of Prevotella.

The study showed that the abundance of Anaerovibrio was significantly higher in CF than that in AF (Table 3). This was probably due to the high-fat content in ration of CF (Supplementary Table S1). Anaerovibrio is a well-known rumen lipolytic bacterium. In the study of microorganisms in rumen of steers, it was found that the relative content of Anaerovibrio in the rumen liquid tended to increase when the oil content in red clover ration increased [27]. In the present study, the Eubacterium content was significantly higher in AF than that in $\mathrm{CF}$, which might be due to the high-fiber content in AF. Eubacterium was mainly involved in the hemicelluloses digestion in the rumen of ruminant and most of the bacteria in this genus could digest xylan [28]. Moreover, it was reported that the abundance of Eubacterium in the rumen of grass-fed cattle was significantly higher than that in the concentrate-fed group [29], which was 


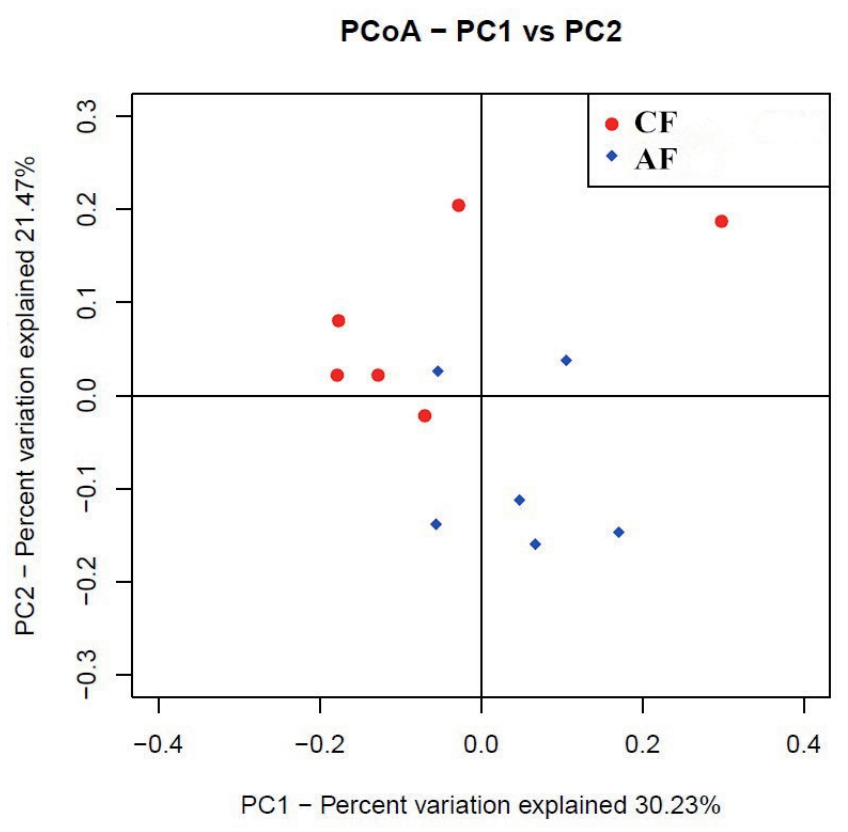

Figure 2. The principal co-ordinate analysis $(\mathrm{PCOA})$ of microbial operational taxomonic units (OTUs) from goats fed a completed feed (CF) or all forage (AF) diet.

in accordance with our results.

\section{CONCLUSION}

The diet had a significant effect on ruminal microbiota in goats. At genus level, the abundance of fiber-, protein-, and fat-digesting bacteria tended to increase with the rise of their corresponding substrate content in the ration. However, the abundance of some genera shared by all samples such as Prevotella remained stable regardless of the changes in ration. They might be the essential microorganisms that maintained normal digestive function of the rumen. Moreover, many unassigned species whose function in the rumen still needs further investigation were identified in our studies.

\section{CONFLICT OF INTEREST}

We certify that there is no conflict of interest with any financial organization regarding the material discussed in the manuscript.

\section{ACKNOWLEDGMENTS}

The authors would like to thank International Cooperation Project of Chinese Ministry of Science and Technology (grant number: 2014DFA32860) for the funding support.

\section{REFERENCES}

1.Flint HJ, Bayer EA, Rincon MT, Lamed R, White BW. Polysaccharide utilization by gut bacteria: Potential for new insights from genomic analysis. Nat Rev Microbiol 2008;6:121-31.

2.Jami E, Israel A, Kotser A, Mizrahi I. Exploring the bovine rumen bacterial community from birth to adulthood. ISME J 2013;7:106979.

3.Bekele AZ, Koike S, Kobayashi Y. Genetic diversity and diet specificity of ruminal prevotella revealed by $16 \mathrm{~s}$ rrna gene-based analysis. FEMS Microbiol Lett 2010;305:49-57.

4.Pitta D, Pinchak W, Dowd S, et al. Longitudinal shifts in bacterial diversity and fermentation pattern in the rumen of steers grazing wheat pasture. Anaerobe 2014;30:11-7.

5.Menezes AB, Lewis E, O'Donovan M, et al. Microbiome analysis of dairy cows fed pasture or total mixed ration diets. FEMS Microbiol Ecol 2011;78:256-65.

6. Wanapat $\mathrm{M}$, Cherdthong A. Use of real-time pcr technique in studying rumen cellulolytic bacteria population as affected by level of roughage in swamp buffalo. Curr Microbiol 2009;58:294-9.

7.Huang XD, Tan HY, Long R, Liang JB, Wright ADG. Comparison of methanogen diversity of yak (bos grunniens) and cattle (bos taurus) from the qinghai-tibetan plateau, china. BMC Microbiol 2012;12:1.

8.Sun Y, Mao S, Zhu W. Rumen chemical and bacterial changes during stepwise adaptation to a high-concentrate diet in goats. Animal 2010;4:210-7.

9.Mohammadzadeh $\mathrm{H}$, Yáñez-Ruiz DR, Martínez-Fernandez G, Abecia L. Molecular comparative assessment of the microbial ecosystem in rumen and faeces of goats fed alfalfa hay alone or combined with oats. Anaerobe 2014;29:52-8.

10. Highlander SK. High throughput sequencing methods for microbiome profiling: Application to food animal systems. Anim Health Res Rev 2012;13:40-53.

11. Caporaso JG, Lauber CL, Walters WA, et al. Global patterns of $16 \mathrm{~s}$ rRNA diversity at a depth of millions of sequences per sample. Proc Natl Acad Sci 2011;108:4516-22.

12. Walters WA, Caporaso JG, Lauber CL, et al. Primerprospector: De novo design and taxonomic analysis of barcoded polymerase chain reaction primers. Bioinformatics 2011;27:1159-61.

13. Caporaso JG, Lauber CL, Walters WA et al. Ultra-high-throughput microbial community analysis on the illumina hiseq and miseq platforms. ISME J 2012;6:1621-4.

14. Edgar RC, Haas BJ, Clemente JC, Quince C, Knight R. Uchime improves sensitivity and speed of chimera detection. Bioinformatics 2011;27:2194-200.

15. Caporaso JG, Bittinger K, Bushman FD, et al. Pynast: A flexible tool for aligning sequences to a template alignment. Bioinformatics 2010; 26:266-267.

16. Caporaso JG, Kuczynski J, Stombaugh J, et al. Qiime allows analysis of high-throughput community sequencing data. Nat Methods 2010; 7:335-6.

17. Benson AK, Kelly SA, Legge R, et al. Individuality in gut microbiota composition is a complex polygenic trait shaped by multiple environmental and host genetic factors. Proc Natl Acad Sci USA 2010;107: 18933-8.

18. Hong PY, Wheeler E, Cann IK, Mackie RI. 2011. Phylogenetic analysis 
of the fecal microbial community in herbivorous land and marine iguanas of the galápagos islands using 16s rRNA-based pyrosequencing. ISME J 5:1461-70.

19. Shanks OC, Kelty CA, Archibeque S, et al. Community structures of fecal bacteria in cattle from different animal feeding operations. Appl Environ Microbiol 2011;77:2992-3001.

20. Jami E, Mizrahi I. Similarity of the ruminal bacteria across individual lactating cows. Anaerobe 2012;18:338-43.

21. Doerner KC, White, BA. Assessment of the endo-1, 4-beta-glucanase components of ruminococcus flavefaciens fd-1. Appl Environ Microbiol 1990;56:1844-50.

22. Shinkai T, Ueki T, Koike S, Kobayashi Y. Determination of bacteria constituting ruminal fibrolytic consortia developed on orchard grass hay stem. J Anim Sci 2014;85:254-61.

23. Huws SA, Lee MR, Muetzel SM, et al. Forage type and fish oil cause shifts in rumen bacterial diversity. FEMS Microbiol Ecol 2010;73: 396-407.

24. Lin HH, Yin LJ, Jiang ST. Cloning, expression, and purification of Pseudomonas aeruginosa keratinase in Escherichia coli AD494 (DE3) plyss expression system. J Agric Food Chem 2009;57:3506-11.

25. Huo W, Zhu W, Mao S. Impact of subacute ruminal acidosis on the diversity of liquid and solid-associated bacteria in the rumen of goats. J Microbiol Biotechn 2014;30:669-80.

26. Koike S, Yoshitani S, Kobayashi Y, Tanaka K. Phylogenetic analysis of fiber-associated rumen bacterial community and PCR detection of uncultured bacteria. FEMS Microbiol Lett 2003;229:23-30.

27. Huws SA, Kim EJ, Lee MR, et al. As yet uncultured bacteria phylogenetically classified as Prevotella, Lachnospiraceae incertae sedis and unclassified Bacteroidales, Clostridiales, and Ruminococcaceae may play a predominant role in ruminal biohydrogenation. Environ Microbiol 2011;13:1500-12.

28. Taguchi H, Koike S, Kobayashi Y, Cann IK, Karita S. Partial characterization of structure and function of a xylanase gene from the rumen hemicellulolytic bacterium Eubacterium ruminantium. J Anim Sci 2004;75:325-32.

29. Tajima K, Aminov R, Nagamine T, et al. Diet-dependent shifts in the bacterial population of the rumen revealed with real-time PCR. Appl Environ Microbiol 2001;67:2766-74. 